\title{
Foreword to the Special Focus on Graph Theory and Applications
}

\author{
Bharati Rajan • Ibrahim Venkat • \\ K. G. Subramanian
}

Published online: 19 April 2016

(C) Springer International Publishing 2016

Graph theory is a fascinating branch of mathematics which has been developed with contributions from Euler, Kirchoff, Cayley, Hamilton, and many others. It is the Swiss mathematician Euler who is credited for having introduced the concept of a graph in solving the famous bridge problem. Many efficient graph algorithms have been developed in the past decade, e.g., for the shortest path problem and the connector problem arising in road and rail networks, the optimal assignment problem in the context of job assigning, and other such problems of practical interest, though there are graph-related problems such as the well-known travelling salesman problem which are NP-complete. The present-day applications of graph concepts and algorithms are in abundance. With an objective to provide a forum for researchers working on problems admitting graph formulations, in well-trodden as well as newer areas, to share their research findings and to benefit from their interactions with experts in the field of graph theory, the School of Computer Science at Universiti Sains Malaysia, Penang organised an International Workshop on Graph Algorithms (IWGA) in May 2015.

The special focus on Graph Theory and Applications in this issue of Mathematics in Computer Science (MCS) contains the revised and enhanced versions of six papers selected from those presented at IWGA 2015. These papers have undergone the usual review-revision process of MCS.

The paper by Bera and Mahalingam explores structural properties of words with a novel approach of relating two apparently different notions: graph and Parikh matrix of a word, a concept that has been introduced recently in the study of numerical properties of words. The authors introduce a new class of graphs, called Parikh word representable graphs, based on the concept of Parikh matrix which provides counts of suitable subwords that are subsequences of a given word. A major result established by the authors is a characterization of these graphs when the words are over a binary alphabet. The authors point out that the problem of characterization is open when the size of the alphabet is greater than two.

\section{B. Rajan}

Department of Mathematics, Loyola College, Chennai 600 034, India

B. Rajan

School of Mathematical and Physical Sciences, Faculty of Science and IT, The University of Newcastle,

Newcastle, NSW 2308, Australia

I. Venkat · K. G. Subramanian $(\bowtie)$

School of Computer sciences, Universiti Sains Malaysia, Penang, Malaysia

e-mail: kgsmani1948@gmail.com 
Construction of codes has been a problem of great interest in coding theory which studies properties of codes for different applications. One of the main results of the paper by Wong is in providing constructions of families of binary linear and nonlinear codes using bipartite graphs.

There has been an increasing interest in the application of graphs in chemistry, in particular to such problems as modelling of organic molecules. The paper by Nagar and Sriram studies connectivity properties of a class of highly branched molecules which are called dendrimers. A parameter called eccentric connectivity index of a graph is found to be useful in the study of properties of molecules considered as graphs. The authors derive formulae for computation of the index for a class of graphs known as eccentric graphs which are defined on the basis of distance-related properties of graphs, in the context of regular dendrimers.

Coloring of graphs is considered as an important issue in graph theory, with the concept of coloring having found use in many application problems. A notion of T-coloring has been formulated on considering certain frequency assignment problems in graph-theoretic terms. The paper by Sivagami and Rajasingh pursues this direction of research and develops methods for computing colorings of graphs, called T-colorings, in the context of certain networks.

Membrane computing is an emerging research field covering computing models inspired by the structure and functioning of living cells. The paper by Mahmood and others deals with one of the main problems of algorithmic graph theory, called minimum dominating set in a graph. A major result of this paper is in providing a linear time algorithm, utilizing the inherent parallelism in P systems, for solving the minimum dominating set problem based on the computing model of $\mathrm{P}$ systems in the area of membrane computing.

Finally, the paper by Sivaselvan and Vijayalakshmi develops an iterative method for the implementation of a Markovian queueing network with multiple closed chains, pointing out the possibility of application in multiple-tier Internet service systems.

The guest editors acknowledge with gratitude the abundant support and guidance received from the MCS managing editor Prof. Dongming Wang. The guest editors would also like to acknowledge the valuable contributions of the reviewers in helping achieve the high quality of the papers and to thank the authors for their contributions.

Another selection of IWGA 2015 papers is planned to appear in special issues of two other journals. 